\title{
The Neurodevelopmental Outcome of Severe Neonatal Haemolytic and Non-hemolytic Hyperbilirubinemia
}

\author{
(1) Amar Taksande \\ Jawaharlal Nehru Medical College, Datta Meghe Institute of Medical Sciences, Department of Pediatrics, Maharashtra, India
}

Keywords: Neonatal, haemolytic, hyperbilirubinemia

Sir,

I have read an interesting study by Çolak et al. (1) published in the June 2020 issue of the Journal of Pediatric Research. The authors studied the neurodevelopmental outcome of severe neonatal haemolytic and nonhemolytic hyperbilirubinemia. The study design, the protocol for conducting the study, was not mentioned in the article. The pivotal aspects of planning a clinical research are the calculations of the sample size and this was not highlighted.

In Table I (1), the Bilirubin/Albumin ratio is given as number (percentage). Although it is statistically insignificant, the value which is given in the haemolytic and non-haemolytic column could not be understood i.e. [3 (21.4\%) vs 9 (40.9\%)].

The author found dehydration in $85.7 \%$ of the nonhaemolytic group as compared to $90.9 \%$ in the haemolytic group of neonates. However, only $7.1 \%$ received IV fluid in the non-haemolytic group as compared to $68.1 \%$ of the haemolytic group. Is there any specific reason for this?
The authors mentioned in the results that 2 neonates develop cerebral palsy (CP) and one neonate had deafness in the haemolytic group. Additionally, they found that 4 neonates received exchange transfusion in the same group. Is there any correlation between the exchange transfusion and the development of CP or deafness in the infants?

To my knowledge, this is the best study of the neurodevelopmental outcomes of severe neonatal hyperbilirubinemia carried out by the author.

\section{Ethics}

Peer-review: External and internal peer-reviewed.

Financial Disclosure: The author has no sources of support for this work.

\section{Reference}

1. ÇolakR, Çalkavurş, YangınErgonE, etal. Theneurodevelopmental outcome of severe neonatal hemolytic and nonhemolytic hyperbilirubinemia. J Pediatr Res 2020; 7:152-7.

\section{Address for Correspondence}

Amar Taksande, Jawaharlal Nehru Medical College, Datta Meghe Institute of Medical Sciences, Department of Pediatrics, Sawangi Meghe, Wardha, Maharashtra, India E-mail: amar.taksande@gmail.com ORCID: orcid.org/0000-0003-3097-7405 Received: 12.06 .2020 Accepted: 28.06 .2020

${ }^{\oplus}$ Copyright 2021 by Ege University Faculty of Medicine, Department of Pediatrics and Ege Children's Foundation The Journal of Pediatric Research, published by Galenos Publishing House. 


\section{Response to the Letter}

\section{Dear Editor,}

We would like to thank you for the opportunity to respond to the issues raised in Dr. Amar Taksande's letter. First of all, the study design was a retrospective observational cohort study. The study started in May 2015 with a file system scan of 36 patients hospitalized due to indirect bilirubinemia over $25 \mathrm{mg} / \mathrm{dL}$ in our Neonatal Intensive Care Unit between June 2013 and January 2015. All patients were contacted via their registered phone numbers whilst in the age range 18-24 months to invite them to our hospital to perform "Bayley Scales of Infant Developmental Assessment scale II" (BSID). We did not perform power analysis since we received all patients who met the inclusion criteria within the specified time interval.

Secondly, the bilirubin/albumin ratio mentioned in Table I shows the number of patients whose bilirubin/albumin ratio should be planned according to the risk category of the baby (1). As stated in the table, there was no statistical difference between the two groups in terms of the number of patients.

Thirdly, we indicate the presence of dehydration if there is a weight loss exceeding physiological limits on that specific postnatal age. However, we do not perform IV fluid replacement for all dehydrated infants in our Clinic of Neonatal Intensive Care Unit if they are vigorous and active, have sufficient oral intake, have normal blood pressure and sufficient urine output. We usually prefer physiological rehydration in such infants by supporting breastfeeding or offering infant formulas. Therefore this difference did not surprise us.

And finally, as seen in Table II, in the hemolysis group, one patient with cerebral palsy and hearing loss who had undergone exchange transfusion, and one patient with only hearing loss who hadn't received exchange transfusion were identified.

Although it is not the best study on this subject and have some limitations, it may be a study with good results in terms of the neurodevelopmental outcomes of severe hyperbilirubinemia.

Thanks you very much for your kind and affirmative comments.

Best regards.

$$
\text { Dr. Rüya Çolak }
$$

University of Health Sciences Turkey, Dr. Behçet Uz Child's Hospital, Clinic of Neonatal Intensive Care Unit, İzmir, Turkey

\section{Reference}

1. American Academy of Pediatrics, Clinical Practice Guideline, Subcommittee on Hyperbilirubinemia. Management of the newborn 35 or more weeks of gestation. Pediatrics 2004; 114: 297-316. 